\title{
Coexistence of classical and quantum plasmonics in large plasmonic structures with subnanometer gaps
}

Kadkhodazadeh, Shima; Wagner, Jakob Birkedal; Kneipp, Harald; Kneipp, Katrin

Published in:

Applied Physics Letters

Link to article, DOI:

$10.1063 / 1.4819163$

Publication date:

2013

Document Version

Publisher's PDF, also known as Version of record

Link back to DTU Orbit

Citation (APA):

Kadkhodazadeh, S., Wagner, J. B., Kneipp, H., \& Kneipp, K. (2013). Coexistence of classical and quantum plasmonics in large plasmonic structures with subnanometer gaps. Applied Physics Letters, 103(8), [083103 ]. https://doi.org/10.1063/1.4819163

\section{General rights}

Copyright and moral rights for the publications made accessible in the public portal are retained by the authors and/or other copyright owners and it is a condition of accessing publications that users recognise and abide by the legal requirements associated with these rights.

- Users may download and print one copy of any publication from the public portal for the purpose of private study or research.

- You may not further distribute the material or use it for any profit-making activity or commercial gain

- You may freely distribute the URL identifying the publication in the public portal 




\section{Coexistence of classical and quantum plasmonics in large plasmonic structures with subnanometer gaps}

Shima Kadkhodazadeh, Jakob B. Wagner, Harald Kneipp, and Katrin Kneipp

Citation: Appl. Phys. Lett. 103, 083103 (2013); doi: 10.1063/1.4819163

View online: http://dx.doi.org/10.1063/1.4819163

View Table of Contents: http://apl.aip.org/resource/1/APPLAB/v103/i8

Published by the AIP Publishing LLC.

Additional information on Appl. Phys. Lett.

Journal Homepage: http://apl.aip.org/

Journal Information: http://apl.aip.org/about/about_the_journal

Top downloads: http://apl.aip.org/features/most_downloaded

Information for Authors: http://apl.aip.org/authors

\section{ADVERTISEMENT}

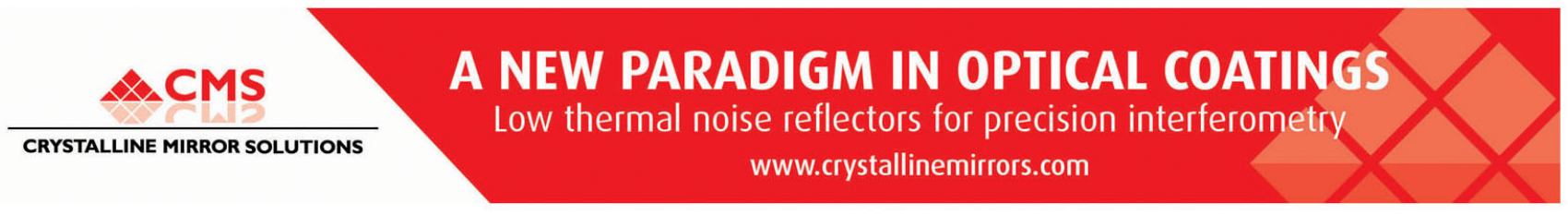




\title{
Coexistence of classical and quantum plasmonics in large plasmonic structures with subnanometer gaps
}

\author{
Shima Kadkhodazadeh, ${ }^{1}$ Jakob B. Wagner, ${ }^{1}$ Harald Kneipp, ${ }^{2}$ and Katrin Kneipp ${ }^{2, a)}$ \\ ${ }^{1}$ Technical University of Denmark, Center for Electron Nanoscopy, Kgs. Lyngby, Denmark \\ ${ }^{2}$ Technical University of Denmark, Department of Physics, Kgs. Lyngby, Denmark
}

(Received 11 June 2013; accepted 7 August 2013; published online 19 August 2013)

\begin{abstract}
Large metal nanostructures with subnanometer interparticle separations (gaps) can provide extremely high local fields and are of particular interest in surface enhanced spectroscopy, as well as for basic understanding of plasmonics. In this experimental electron energy loss study, we monitor the transition of plasmonic dimers from a classical to a quantum system by decreasing gaps to dimensions when tunneling occurs and a conductive nanobridge evolves. Our studies show that silver dimers with atomic scale gaps can exhibit a regime, in which charge transfer plasmon modes, as a hallmark of a quantum nature, and "classical" bright and dark dipolar plasmon modes can be seen simultaneously. (C) 2013 AIP Publishing LLC. [http://dx.doi.org/10.1063/1.4819163]
\end{abstract}

Aggregates of metal nanoparticles with dimensions in the order of tens of nanometers and with subnanometer interparticle gaps can generate highly confined and enhanced local fields, opening up exciting capabilities for photondriven processes. ${ }^{1,2}$ They provide the highest enhancement level obtained in plasmon supported spectroscopy so far and enable, for example, the detection of Raman signals of single molecules. ${ }^{3,4}$ Moreover, metal nanostructures with subnanometer gaps are of basic interest as plasmonic systems, which can exhibit a quantum nature for subnanometer gaps due to electronic density spill out and tunneling. ${ }^{5}$ The onset of the quantum regime is marked with the appearance of the charge transfer plasmon (CTP) modes for closely spaced or touching nanoparticles. However, studying these structures experimentally has been a challenge due to the difficulties involved in creating and manipulating subnanometer interparticle distances. Current state-of-the-art fabrication processes are capable of producing plasmonic structures in sub-nanometer regime. ${ }^{6}$ Methodological developments for probing plasmonic systems at high energy and spatial resolution with photons and electrons, ${ }^{7}$ in particular non-linear effects, ${ }^{8}$ can enable us to "see" quantum and non-local effects. Recently, two experimental observations of quantum tunneling between plasmonic nanostructures have been reported, one involving simultaneous optical and electrical measurements across two gold coated atomic force microscopy tips ${ }^{9}$ and the other involving electron energy loss spectroscopy (EELS) measurements of two approaching silver nanospheres in a transmission electron microscope (TEM). ${ }^{10}$

The choice of EELS for characterization of plasmonic nanostructures has become increasingly popular, due to the subnanometer spatial resolution of this technique. ${ }^{11,12}$ Depending on the geometry of the plasmonic structure and the position of the electron beam, EELS can probe bright and/or dark dipole modes and higher order modes. ${ }^{13}$ Therefore, the same optically active dipolar resonances, which dominate the optical response of plasmonic structures,

\footnotetext{
${ }^{\text {a) }}$ Author to whom correspondence should be addressed. Electronic mail: katrin.kneipp@fysik.dtu.dk
}

can be excited and studied by the electron beam in EELS. Moreover, it has been demonstrated that the movement of small nanoparticles can be manipulated by the electron probe, enabling Ångstrom scale movements in single or multiple nanoparticles. ${ }^{14}$ This effect was exploited to record EELS spectra of two approaching silver nanoparticles and observe the appearance and evolution of charge transfer plasmon modes. ${ }^{10}$ Here, we have studied surface plasmons in silver dimers with decreasing gaps to monitor the transition in the plasmonic response of these structures from a classical to a quantum regime. In particular, we explore gradual changes in the plasmon spectrum for gaps of atomic dimensions, when tunneling between the silver spheres occurs and, for further decreasing gaps, where a conductive nanobridge is formed between them.

The silver nanoparticles studied were prepared by laser ablation. $532 \mathrm{~nm}$ laser pulses with pulse lengths of $10 \mathrm{~ns}$, a repetition rate of $10 \mathrm{~Hz}$, and pulse energies of $\sim 0.15 \mathrm{Ws}$ were focused in a spot of $\sim 0.5 \mathrm{~mm}$ onto a silver foil in deionized water. The resulting silver nanospheres were deposited on a $5 \mathrm{~nm}$ thick SiN membrane for EELS experiments. The EELS spectra were acquired in scanning TEM (STEM) mode and with the instrument operated at $120 \mathrm{kV}$. The spatial and energy resolutions were approximately $3-4 \AA$ and $0.15 \mathrm{eV}$, respectively. High angle annular darkfield (HAADF) STEM images of the dimers were recorded before and after each EELS acquisition, in order to measure the position of the particles and their gap size. In HAADF STEM, the image is considered to be a convolution of the electron probe profile and the object. ${ }^{15}$ Given that here gap sizes comparable to the size of the electron probe are measured, it becomes necessary to deconvolute the contribution of the probe from the images, in order to measure distances below $1.0 \mathrm{~nm}$ more accurately. This was done by assuming a Gaussian profile with a full width half maximum of $4 \AA$ for the electron probe to perform the deconvolution. We estimate a $\pm 2 \AA$ error in the measurements.

The ablation process delivers isolated silver nanoparticles and small aggregates. HAADF STEM images of an isolated silver sphere and two spheres forming a dimer are 
(a)

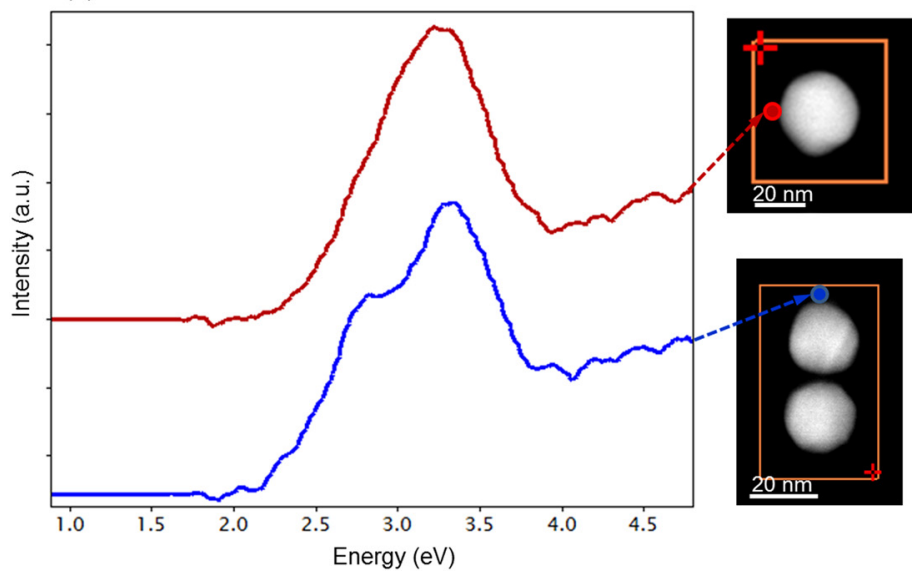

shown in Figure 1(a). The corresponding EELS spectra of the structures demonstrate how the dipolar surface plasmon resonance in a single particle around $3.22 \mathrm{eV}$ is hybridized into the antibonding dipolar plasmon (ADP) mode (also known as dark mode) around $3.30 \mathrm{eV}$ and the bonding dipolar plasmon (BDP) mode (also known as bright mode) around $2.75 \mathrm{eV}$ in a dimer, as the result of interparticle coupling. ${ }^{16}$ EELS intensity maps of the corresponding plasmon resonances for an isolated sphere and a dimer are shown in Figure 1(b). The maps confirm the mode assignments: as expected, the dark mode is excited much more strongly than the bright mode, when the electron beam is placed in the gap between the particles. ${ }^{13,17}$

Coupling between the particles is expected to increase as the distance between the particles is reduced, producing a redshift in the BDP resonance. ${ }^{18,19}$ Our EELS measurements from different silver dimers with gap sizes varying between 7.0 and $1.0 \mathrm{~nm}$ are displayed in Figure 2. The measurements show a continuous redshift of the BDP mode as the interparticle gap is decreased. The redshift of the BDP mode with decreasing distance between nanospheres has been explained by classical electromagnetic models using the local response function. ${ }^{18}$ This effect is exploited, for example, in the

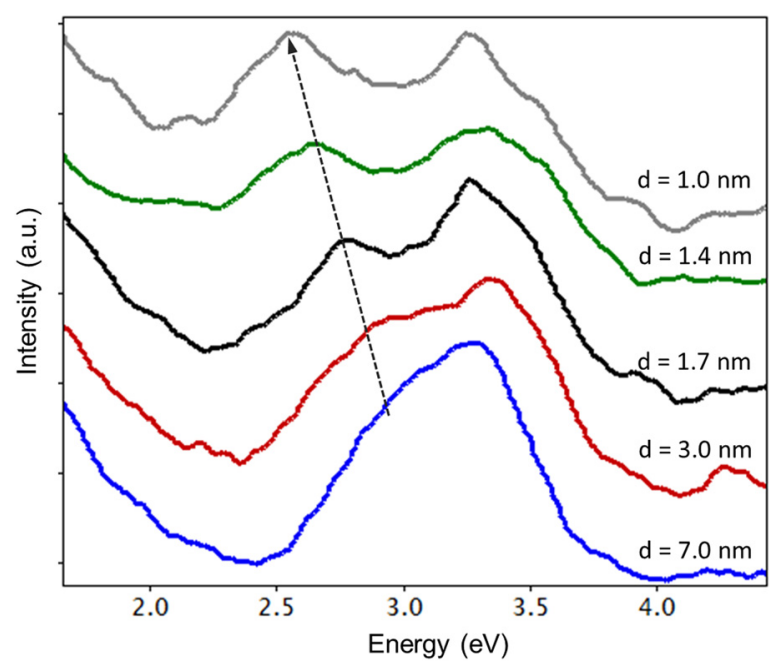

FIG. 2. Redshift of bonding dipolar plasmon modes for decreasing gaps in "classical" plasmonic dimers: EELS spectra collected from dimers formed by silver spheres of 20-25 nm diameter and varying gaps from 7.0 to $1.0 \mathrm{~nm}$. The electron beam was positioned in the periphery of the dimer in each case. (b)

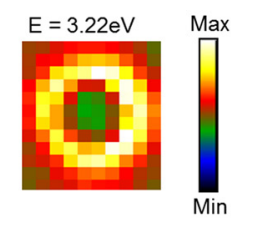

48
FIG. 1. Plasmon resonances of an isolated sphere and a dimer due to plasmon hybridization: (a) HAADF STEM images of an isolated silver sphere and two spheres forming a dimer along with their electron energy loss spectra displaying the plasmon resonances for each structure. (b) EELS intensity maps of the plasmon modes obtained by integrating the intensity of the EELS spectra at each pixel within a $0.2 \mathrm{eV}$ wide energy window. context of plasmonic rulers. ${ }^{20-23}$ Additionally, non-local effects have to be incorporated in order to describe the plasmonic response of these structures more accurately. ${ }^{24,25}$ In general, to understand the plasmonic response of structures with atomic size interparticle gaps, quantum effects have to be incorporated. ${ }^{26}$ Such narrow-gap structures are on the other hand of considerable interest for enhanced optical and spectroscopic effects.

A closer examination of the evolution of the surface plasmon modes of two silver spheres beyond their classical local response was performed by using the electron probe to induce an attractive motion between two closely spaced silver spheres. Spectra A-C in Figure 3 correspond to the EELS spectra from the silver spheres when the gap is reduced from $1.0 \mathrm{~nm}$ down to $-0.6 \mathrm{~nm}$. A negative gap denotes overlap between the particles. Spectrum A with $\mathrm{d}=1.0 \mathrm{~nm}$ in Figure 3 exhibits the BDP and ADP modes, typical of a classical behavior, and resembling the spectra of other silver dimers in Figure 2. As the gap between the silver particles is reduced to $0.3 \mathrm{~nm}$ in spectrum $\mathrm{B}$, the BDP and ADP modes are still present and the BDP appears to have redshifted from $2.68 \mathrm{eV}$ to $2.40 \mathrm{eV}$ compared to spectrum A. This is signature of a classical behavior. Furthermore, an additional peak is observed in spectrum $\mathrm{B}$ around $2.9 \mathrm{eV}$ with $\mathrm{d}=0.3 \mathrm{~nm}$, a dimension where tunneling is expected to start to play a role. The appearance of this mode is in agreement with theoretical calculations in the framework of a quantum corrected classical model. ${ }^{5}$ The onset of this plasmon mode before the particles are in contact strongly suggests that this mode is related to the onset of the tunnel current. As the particles continue to approach, they make contact and a bridge develops between them. In spectrum $\mathrm{C}$ with $\mathrm{d}=-0.6 \mathrm{~nm}$, an additional mode is present at $1.21 \mathrm{eV}$. The appearance of this mode after contact between the particles is in agreement with both reported theoretical and experimental results ${ }^{5,10}$ and is described to be a CTP mode related to an oscillating current between the two spheres. The tunneling CTP at around $2.9 \mathrm{eV}$ can also be seen in spectrum C. However, it appears that the BDP mode is no longer present at this stage. Theoretically, a higher energy CTP has also been described for such a dimer system. ${ }^{5}$ Since the classical dipolar modes break down after the particles come in contact, it is likely that the mode around $3.5 \mathrm{eV}$ is the third CTP mode predicted in theoretical studies. 


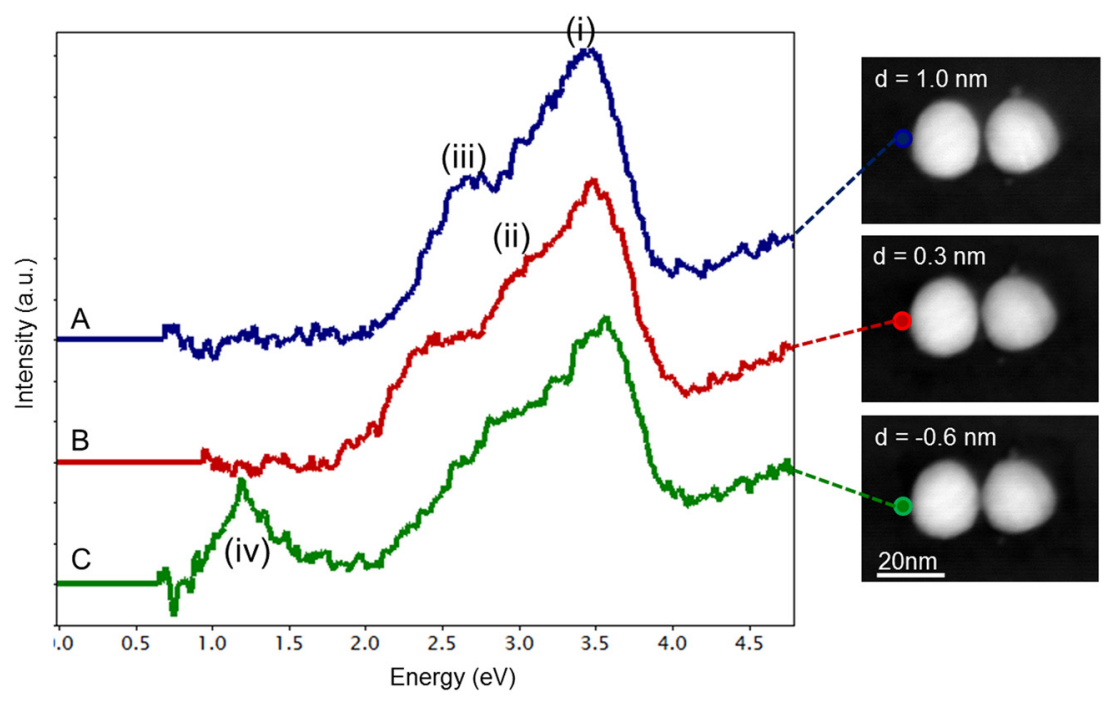

FIG. 3. STEM images and EELS spectra from silver dimers in non-contact-, tunnel-, and contact-regimes collected from the same dimer but with decreasing gaps. Spectra A, B, and C represent the non-contact-, tunnel-, and contactregimes, respectively. The bright (iii), dark modes (i) and a charge transfer plasmon mode (ii) related to the tunnel current are marked in the spectra. The circle indicates the position of the electron beam. Spectrum B shows the coexistence of the classical and quantum related plasmons in the dimer.

We now consider in more detail the plasmonic response of a dimer as the gap narrows down to atomic dimension and beyond, when a nanobridge begins to evolve between the particles. The spectra in Figure 4(a) are collected from a dimer with $\mathrm{d}=0.2 \mathrm{~nm}$ down to $\mathrm{d}=-0.9 \mathrm{~nm}$. Different plasmon modes appearing in the spectra are labeled with numbers (i)-(iv) and the energy and intensity of each feature for different gap distances are summarized in Figure 4(b). The energy and intensity values were inferred from least square fitting of the spectrum with each feature estimated as a Gaussian function. Peaks (i), (ii), and (iii) in spectrum A of Figure 4(a) correspond to the ADP, CTP related to the onset of tunneling and BDP modes, respectively. Consistent with our observation in Figure 3 spectrum B, the bright and dark dipolar modes continue to exist even after the onset of a CTP mode related to tunneling. As the particles come in contact in spectrum B, the BDP disappears. In contrast, peak (i), previously attributed to the ADP mode, appears to continue to exist also in the contact regime. As explained previously, this mode can be a higher energy CTP mode appearing at a similar energy to the ADP mode. Two additional CTP modes, (ii) and (iv), can be distinctly seen in spectrum B, where mode (ii) is related to tunneling and (iv) related to current flow through the nanobridge formed between the particles. The latter CTP mode has also been experimentally observed for two gold nanoprisms connected by a gold bridge. ${ }^{6}$ As it has been discussed in the literature, the transition from a non-contact tunneling regime to a full contact regime is not sharp, but gradual. ${ }^{5}$ The graphs in Figure 4(b) illustrate this evolution by plotting the energy and intensity of modes (i)-(iv) as a function of the interparticle gap in spectra A-D. As a wider bridge develops between the particles in spectra B-D, CTP mode (iv) blueshifts from 1.21 to $1.46 \mathrm{eV}$ and its intensity increases. This is due to the increase of current flow between the particles. In contrast, the intensity of the tunneling related mode (ii) decreases with increasing contact area and current flow between the particles. Further decreasing the gap in the dimer leads to a complete merging of the nanospheres and results in return to a single "classical"


FIG. 4. EELS spectra collected from a dimer during the transition from tunneling to a nanocontact regime: (a) STEM image and EELS spectra collected when the gap narrows down from $d=0.2 \mathrm{~nm}$, including the contact regime $(\mathrm{d}<0)$. The circle in the STEM image indicates the position of the electron beam. Spectrum A represents the tunneling regime, where the classical dark (i) and bright (iii) modes coexist along with a tunneling related CTP mode (ii). After the particles make contact in spectra B-D, a second lower energy CTP mode (iv), related to current flow between the particles appears. Mode (i) is now likely to be a third CTP mode. The energy and intensity of modes (i)-(iv) are plotted in (b), demonstrating how different modes evolve with increasing contact area between the particles. 


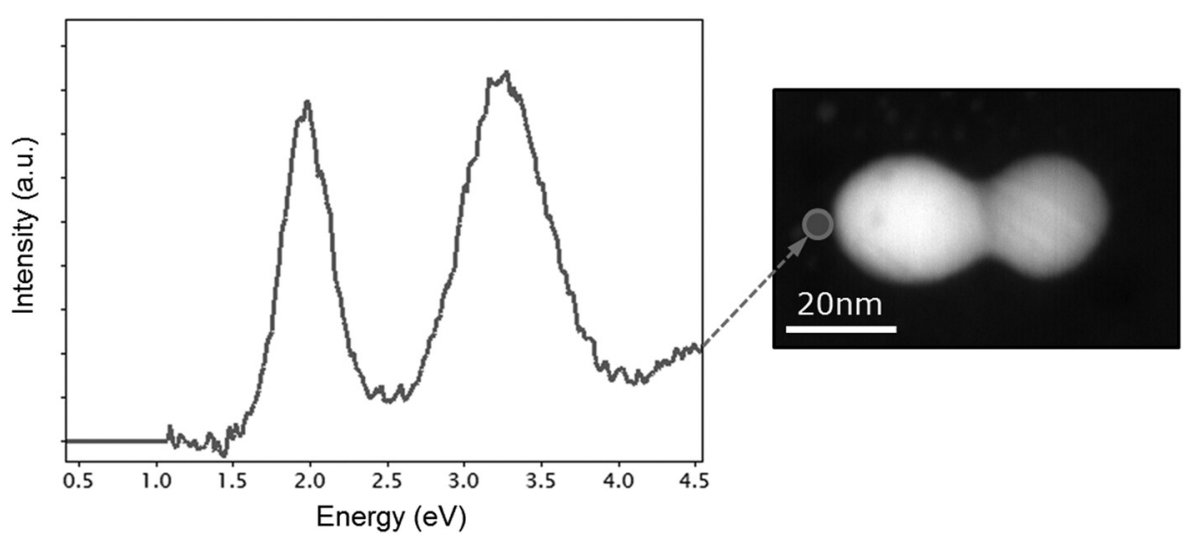

FIG. 5. STEM image and EELS spectrum of two merged spheres, behaving as a classical elongated nanoparticle. The EELS spectrum displays only the dipole mode and a higher order mode of the plasmonic structure.

elongated nanoparticle. Such a structure displays dipolar and higher order plasmon modes, as shown in Figure 5.

In conclusion, by employing EELS, we have studied the plasmon modes in dimers formed of silver spheres ca. $20 \mathrm{~nm}$ in diameter and with narrow gaps. By exploiting such structures with decreasing gaps down to atomic dimension, we can generate a controlled transition from a classical to a quantum plasmonic system and back to a classical system. We identified a quantum regime of plasmonic resonances, when tunneling occurs at atomic dimension gaps and in which charge transfer plasmon modes and bonding dipolar plasmon modes are seen to be simultaneously supported by the same structure. Further decreasing the gap in the dimer, i.e., merging the nanospheres, results in returning to single "classical" elongated nanoparticle. The preservation of classical dipolar modes in the quantum regime when tunneling occurs can have important implications for local fields of "large" plasmonic nanostructures in subnanometer gaps. It might imply that the local fields do not collapse in such small gaps. On the other hand, a non-local behavior of the classical mode when the gap narrows down to atomic scale might result in a decrease of local optical field intensity by about one order of magnitude compared with the prediction based on local dielectric response. ${ }^{24}$

The A. P. Møller and Chastine Mc-Kinney Møller Foundation are gratefully acknowledged for their contribution towards the establishment of the Centre for Electron Nanoscopy in the Technical University of Denmark.

${ }^{1}$ M. I. Stockman, Opt. Express 19, 22029 (2011).

${ }^{2}$ N. J. Halas, S. Lal, W. S. Chang, S. Link, and P. Nordlander, Chem. Rev. 111, 3913 (2011).

${ }^{3}$ K. Kneipp, W. Yang, H. Kneipp, L. T. Perelman, I. Itzkan, R. R. Dasari, and M. S. Feld, Phys. Rev. Lett. 78, 1667 (1997).
${ }^{4}$ H. X. Xu, E. J. Bjerneld, M. Kall, and L. Borjesson, Phys. Rev. Lett. 83, 4357 (1999).

${ }^{5}$ R. Esteban, A. G. Borisov, P. Nordlander, and J. Aizpurua, Nature Commun. 3, 825 (2012).

${ }^{6}$ H. G. Duan, A. I. Fernandez-Dominguez, M. Bosman, S. A. Maier, and J. K. W. Yang, Nano Lett. 12, 1683 (2012).

${ }^{7}$ F. J. G. de Abajo, Rev. Mod. Phys. 82, 209 (2010).

${ }^{8}$ A. Yurtsever, R. M. van der Veen, and A. H. Zewail, Science 335, 59 (2012).

${ }^{9}$ K. J. Savage, M. M. Hawkeye, R. Esteban, A. G. Borisov, J. Aizpurua, and J. M. Baumberg, Nature 491, 574 (2012).

${ }^{10}$ J. A. Scholl, A. Garcia-Etxarri, A. L. Koh, and J. A. Dionne, Nano Lett. 13, 564 (2013).

${ }^{11}$ J. Nelayah, M. Kociak, O. Stephan, F. J. G. de Abajo, M. Tence, L. Henrard, D. Taverna, I. Pastoriza-Santos, L. M. Liz-Marzan, and C. Colliex, Nat. Phys. 3, 348 (2007).

${ }^{12}$ M. W. Chu, V. Myroshnychenko, C. H. Chen, J. P. Deng, C. Y. Mou, and F. J. G. de Abajo, Nano Lett. 9, 399 (2009).

${ }^{13}$ A. L. Koh, K. Bao, I. Khan, W. E. Smith, G. Kothleitner, P. Nordlander, S. A. Maier, and D. W. McComb, ACS Nano 3, 3015 (2009).

${ }^{14}$ P. E. Batson, A. Reyes-Coronado, R. G. Barrera, A. Rivacoba, P. M. Echenique, and J. Aizpurua, Nano Lett. 11, 3388 (2011).

${ }^{15}$ S. J. Pennycook and D. E. Jesson, Proc. R. Soc. London, Ser. A 441, 261 (1993).

${ }^{16}$ P. Nordlander, C. Oubre, E. Prodan, K. Li, and M. I. Stockman, Nano Lett. 4, 899 (2004).

${ }^{17}$ N. Zabala, A. Rivacoba, and P. M. Echenique, Phys. Rev. B 56, 7623 (1997).

${ }^{18}$ H. X. Xu and M. Kall, Surface-Enhanced Raman Scattering: Physics and Applications (Springer-Verlag, Berlin, 2006), Vol. 103, p. 87.

${ }^{19}$ I. Romero, J. Aizpurua, G. W. Bryant, and F. J. G. de Abajo, Opt. Express 14, 9988 (2006).

${ }^{20}$ K. H. Su, Q. H. Wei, X. Zhang, J. J. Mock, D. R. Smith, and S. Schultz, Nano Lett. 3, 1087 (2003).

${ }^{21}$ L. Gunnarsson, E. J. Bjerneld, H. Xu, S. Petronis, B. Kasemo, and M. Kall, Appl. Phys. Lett. 78, 802 (2001).

${ }^{22}$ P. K. Jain and M. A. El-Sayed, Chem. Phys. Lett. 487, 153 (2010).

${ }^{23}$ L. L. Yang, H. Y. Wang, B. Yan, and B. M. Reinhard, J. Phys. Chem. C 114, 4901 (2010).

${ }^{24}$ F. J. G. de Abajo, J. Phys. Chem. C 112, 17983 (2008).

${ }^{25}$ G. Toscano, S. Raza, A. P. Jauho, N. A. Mortensen, and M. Wubs, Opt. Express 20, 4176 (2012).

${ }^{26}$ J. Zuloaga, E. Prodan, and P. Nordlander, ACS Nano 4, 5269 (2010). 